\title{
Tunneling vortices induce quantized responses in exciton-condensate/weak excitonic condensate/exciton-condensate (EC/EC'/EC) junctions
}

\author{
Yi-Ting Tseng, Yen-ju Wu, Ya-Fen Hsu, and Jung-Jung Su* \\ Department of Electrophysics, National Yang Ming Chiao Tung University, Hsinchu 300, Taiwan
}

(Received 3 December 2020; revised 3 December 2021; accepted 7 December 2021; published 4 March 2022)

\begin{abstract}
Junctions composed of excitonic condensate (EC) bilayers exhibit intriguing physics due to the presence of interlayer tunneling. Together with the excitonic superflow, this tunneling induces a special kind of topological object which is analogous to the Josephson vortices in extended superconducting Josephson junction. We name such objects the "tunneling vortices." In this work we propose to characterize the topological properties of these vortices in the current-injecting scheme under which multiple vortices can easily be introduced through current injection from one end of the EC junctions. We find theoretically that current responses demonstrate the quantized nature of vortices: the ejected currents show periodicity as a function of injected currents(or vortex numbers). Moreover, the injected-ejected current difference shows plateaus corresponding to different vortex numbers when varying tunneling strength $\Delta_{t}$ or superfluid density $\rho_{S}$. Since all parameters are experimentally accessible, we expect real-world demonstrations of these enthralling quantization behaviors to come in the near future.
\end{abstract}

DOI: 10.1103/PhysRevResearch.4.013177

\section{INTRODUCTION}

Excitons, the electron-hole pairs bound by Coulomb interaction, have never failed to amaze us physicists with phenomena that only occur when they reach the state of spontaneous phase coherence-the excitonic condensate [1,2]. Bilayer structures are then designed to attain such condensates by separating the constituent electrons and holes of excitons in two closely spaced layers, extending the exciton lifetime substantially. The bilayer structures also make possible for electrical currents of the constituent electrons and holes to be probed and be controlled individually [3]. Related electrical transport, including the coherent interlayer tunneling [4-10], the quantum Hall drag [11-14], and the counterflow supercurrents $[15,16]$, have been intensively studied in quantum Hall bilayer [17-20] of GaAs starting at the beginning of the century. In the last decades, vast attention has also been drawn to bilayers of van der Waals materials [21-29], mainly that of the graphene base. Their even greater electrical accessibility and smaller layer separations allow for potentially high-temperature condensates.

Originating from the nature of bilayers, interlayer tunneling gives exciting twists to the excitonic condensation phenomena. Especially when collaborating with exciton superflow, it can give rise to topologically robust objects. Such topological objects are governed by sine-Gordon physics and

\footnotetext{
*jungjsu@nctu.edu.tw

Published by the American Physical Society under the terms of the Creative Commons Attribution 4.0 International license. Further distribution of this work must maintain attribution to the author(s) and the published article's title, journal citation, and DOI.
}

are analogous to the Josephson vortices [30] in superconducting Josephson junctions or even the dynamics of the pendulum [31]. Here we name this object type the "tunneling vortex." We study this type of vortices specifically in the so-called "EC/EC'/EC" junctions [32-35], junctions composed of two ideal excitonic condensates (ECs) sandwiching a weak condensate EC' with controllable length. In our previous work [36-39], a tunneling vortex can be created by imposing a condensate phase externally to part of the system, and the vortex will be pinned right at the phase boundary. When the external phase is not an integer multiple of $2 \pi$, it can even induce vortices that possess fractional charge, resembling the fractional vortices [40-45] in $0-\kappa$ superconducting Josephson junctions.

In this work we explore a dimension of tunneling vortices in EC/EC'/EC junctions by employing current injection for vortex generations. This method facilitates the generation of multiple vortices which align themselves as a train. A similar "train" of sine-Gordon-governed topological objects includes a soliton lattice in a nearly commensurate charge density wave $[46,47]$ in solids, coupled pendula [31], and the "flux-flow oscillators" [48-59] in the "flux-flow" regime in extended superconducting Josephson junctions. The flux-flow oscillators, for example, can be created by a uniform magnetic field application [48-52] or by local current injections [60-67]. Our current-injection scheme actually resembles more of the former in the sense that it creates a nearly uniform phase gradient over the system. That phase gradient produces in space a $2 \pi$ phase span, which prompts tunneling current of opposite directions and creates an electron current circulation-the tunneling vortex (lower panel of Fig. 1). The tunneling current also modifies the phase profile in return so that instead of being a constant gradient (black dash line), the phase profile now bends into the curve shown as the full red line. The 


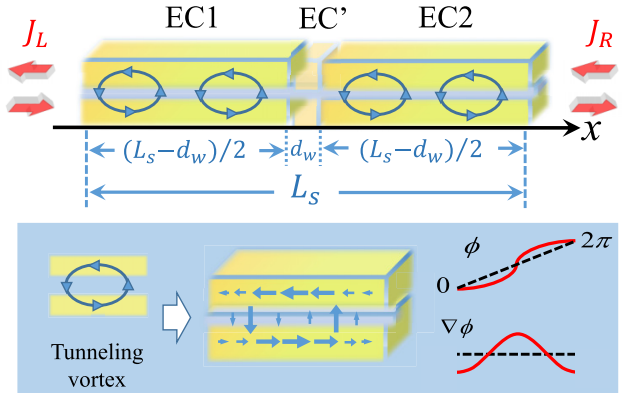

FIG. 1. Illustration of tunneling vortices formation in EC/EC'/ EC junction under the current-injecting scheme. (Upper) The counterflow current $J_{L}$ is injected from the left, and ejected current $J_{R}$ is measured in the right. The injected current induces tunneling vortices. (Lower) Tunneling currents in opposite directions together with counterflow supercurrent form a vortex. In the presence of interlayer tunneling, the phase profile bands from black dashed line (no tunneling) to red solid line that has a maximum tangent (most significant supercurrent density) at the center and minimum tangent at $\phi=0$ and $\phi=2 \pi$.

corresponding phase gradient (essentially the supercurrent density) correspondingly exhibits a ripple instead of horizontal.

The ripple in the supercurrent density can be detected when a tunneling vortex passes the other end. However, as required by the sine-Gordon equation, the ripple size is set by the interlayer tunneling strength, which can be small in a decent condensate sample. In this work we find a way to go beyond the sine-Gordon physics and enlarge the current response of vortex creation. To do that we need a sufficiently long weak condensate area (EC') in the EC/EC'/EC junction to induce the tunneling-assisted Andreev reflection (TAAR) [38] process inside. That way, not all electrons in EC' are in the Andreev bound state but only part of them travel within EC' through interlayer tunneling. From the authors' previous work [38], when TAAR happens, the average supercurrent in the left (EC1) and the right EC (EC2) can be different while the local current conservation remains. That means that the left-right supercurrent-density difference can then add to the original ripples to enhance the vortex response. Notice that TAAR is beyond the sine-Gordon physics and results from our pseudospin Landau-Lifshitz-Gilbert (LLG) calculation. While the LLG equation can be simplified to the stationary sineGordon equation in the time-independent limit of a perfect condensate (with no charge imbalance), it can demonstrate the physics beyond - the enhanced vortices quantization behavior in $\mathrm{EC} / \mathrm{EC}$ '/EC junctions.

\section{PSEUDOSPIN LANDAU-LIFSHITZ-GILBERT (LLG) EQUATION}

We use the pseudospin Landau-Lifshitz-Gilbert equation [36-39,68] to describe the electrical transport in our excitonic condensate systems. This approach has proven to be most effective yet reliable in describing experimental results $[8,10,39]$. It is detailed in the authors' previous works [36-38], and here we only guide the readers briefly through necessary steps for the current discussion.
This method starts by mapping the condensate's local $\mathrm{SU}(2)$ degree of freedom to an $\mathrm{O}(3)$ vector, usually referred to as the "pseudospin." In this language the local pseudospin vectors are determined by two quantities - the vector's $z$ component $m_{z}$ and its azimuthal angle $\phi$. The former corresponds to the population difference between the two layers, the later the phase of its condensate wave function.

The dynamics of the pseudospin is described by the LLG equation,

$$
\frac{d \vec{m}[X]}{d t}=\vec{m} \times \vec{H}_{p s}[X]-\alpha\left(\vec{m}[X] \times \frac{d \vec{m}[X]}{d t}\right),
$$

where $X$ indicates the location of pseudospins and $\alpha$ the Gilbert damping parameter. From this equation we see that the steady-state physics is essentially governed by the pseudospin magnetic field $\vec{H}_{p s} \equiv(2 / n \hbar)(\delta E[\vec{m}] / \delta \vec{m})$, with the system's energy functional:

$$
\begin{aligned}
E[\vec{m}]=L_{x} L_{y} \cdot \sum_{X} & {\left[\beta m_{z}^{2}+\frac{\rho_{S}[X] m_{\perp}^{2}}{2}\left|\nabla_{X} \phi\right|^{2}\right.} \\
& \left.-\frac{n \Delta_{t} m_{\perp}}{2} \cos \phi\right] .
\end{aligned}
$$

The first term accounts for the energy cost for charge imbalance between layers and is characterized by the anisotropy parameter $\beta$. The second term measures the energy cost to wind the condensate phase $\phi$ with the superfluid density $\rho_{S}$. The third term describes single-particle interlayer tunneling, with $n$ being density and $\Delta_{t}$ the tunneling strength. This phenomenological model is originally derived microscopically using mean-field theory and has been justified by experiments $[8,39]$

With the pseudospin magnetic field obtained by $E[\vec{m}]$ along with the dissipation term, we arrive at the LLG equation for pseudospins:

$$
\begin{gathered}
\dot{m}_{z}=\left\{-\frac{2}{n \hbar} \rho_{S} m_{\perp}^{2} \vec{\nabla}^{2} \phi+\frac{\Delta_{t}}{\hbar} m_{\perp} \sin \phi\right\}+\alpha_{z} m_{\perp}^{2} \dot{\phi}, \\
\dot{\phi}=m_{z}\left\{\frac{2}{n \hbar} \rho_{S}\left(|\vec{\nabla} \phi|^{2}+\frac{2}{m_{\perp}^{4}}\left|\vec{\nabla} m_{z}\right|^{2}+\frac{2 m_{z}}{m_{\perp}^{2}} \vec{\nabla}^{2} m_{z}\right)\right. \\
\left.\times \frac{4}{n \hbar} \beta-\frac{\Delta_{t}}{\hbar} \frac{1}{m_{\perp}} \cos \phi\right\}-\frac{\alpha_{\phi}}{m_{\perp}^{2}} \dot{m}_{z} .
\end{gathered}
$$

We now tailor the above formulation to model our currentinjecting EC-EC'-EC system. In the discussion below we assume translationally invariant in the $y$ direction, the direction in the layer plane but perpendicular to the transport direction. Our system consists of two long ideal excitonic condensates, EC1 and EC2, sandwiching a weak condensate (EC'), which has a much shorter length $d_{w}$. The weak condensate is characterized by a lesser superfluid density $\rho_{\mathrm{Sw}}$, namely,

$$
\rho_{S}(x)= \begin{cases}\rho_{S 0}, & -L_{s} / 2<x<-d_{w} / 2, d_{w} / 2<x<L_{s} / 2 \\ \rho_{\mathrm{Sw}}, & -d_{w} / 2<x<d_{w} / 2,\end{cases}
$$

where $L_{S}$ is the length of the system. 
Next we impose injected current $j_{L}$ by the following boundary conditions:

$$
\left.\frac{e \rho_{S}}{\hbar} \nabla \phi\right|_{x=-L_{s} / 2}=j_{L},\left.\quad \nabla^{2} \phi\right|_{x=-L_{s} / 2}=0 .
$$

The first one represents the current injection of $j_{L}$ from the left. It is expressed as a supercurrent contribution because the normal external current can fully transform into supercurrent through Andreev reflection immediately at the contact [15]. The second term requires that there be no supercurrent loss at the left boundary, meaning that the tunneling current has to be diminished to zero [39]. It has been shown that both injected current and tunneling current at the boundary are controllable experimentally $[8,10]$. With this geometrical information, we then solve the LLG equation numerically by slowly sweeping the injected current density $j_{L}$ and see the current response $j_{R}$ and the spatial profiles of phase and current.

\section{TUNNELING VORTICES IN EC/EC'/EC JUNCTIONS}

In this section we summarize our results of calculation and show how the discretized nature of the vortices plays decisive roles on the current response in the EC/EC'/EC junctions. The parameters used in this work are $\beta=0.02 E_{0}$, $\rho_{S 0}=0.005 E_{0}, \rho_{\mathrm{Sw}}=0.0005 E_{0}$, and $\Delta_{t}=10^{-6} E_{0}$, where $E_{0}$ is the characteristic energy scale of the system, the Coulomb energy. Taking the most tested system, quantum Hall bilayer, as an example, $E_{0} \equiv e^{2} / \epsilon l \sim 7 \mathrm{meV}$, where $l \sim 18 \mathrm{~nm}$ [2] is the magnetic length [69]. The values we used for $\beta$ and $\rho_{s 0}$ were derived from the mean-field calculation. The tunneling strength $\Delta_{t}$ usually ranges from $10^{-8} E_{0}$ to $10^{-6} E_{0}$ in the current active systems according to the experimental reports $[7,70]$. Here we use the larger allowed value $\left(\Delta_{t}=10^{-6} E_{0}\right)$ unless otherwise specified; this larger value can better demonstrate the vortices effect. The length of the system used is $L_{s}=400 l \sim 7.2 \mu \mathrm{m}$ in the absence of EC'. Otherwise, $d_{w}=30 l \sim 0.54 \mu \mathrm{m}$ and $L_{s}=(400+30) l \sim 7.74 \mu \mathrm{m}$. The length of the system does not play a crucial role in this work. Generally, at the same injected current level, more vortices are introduced in a longer system. We therefore define a current unit,

$$
j_{0}=\frac{e \rho_{S}}{\hbar} \frac{2 \pi}{L_{s}} .
$$

Interpreting directly from this expression, $j_{0}$ represents the current required to produce an additional $2 \pi$ phase winding in the absence of tunneling. It turns out that $j_{0}$ also yields the current density to introduce one more tunneling vortex into the system, as we will show later. Lastly, all of our results are obtained by gradually increasing the injected current from zero at an increased rate of $4 \times 10^{-7}\left[j_{0} /\left(h / E_{0}\right)\right]$.

We start our numerical investigation from fundamental behaviors in the current-injected scheme in the absence of the weak condensate. Figure 2 shows the phase profile and the supercurrent-density profile for the case of $j_{L} / j_{0}=5.95$ (and no EC'). Looking at the phase profile (upper panel of Fig. 2) with bare eyes, it appears to be a straight line. However, the corresponding supercurrent-density profile (essentially the phase profile's spatial derivative) shows ripples. These ripples

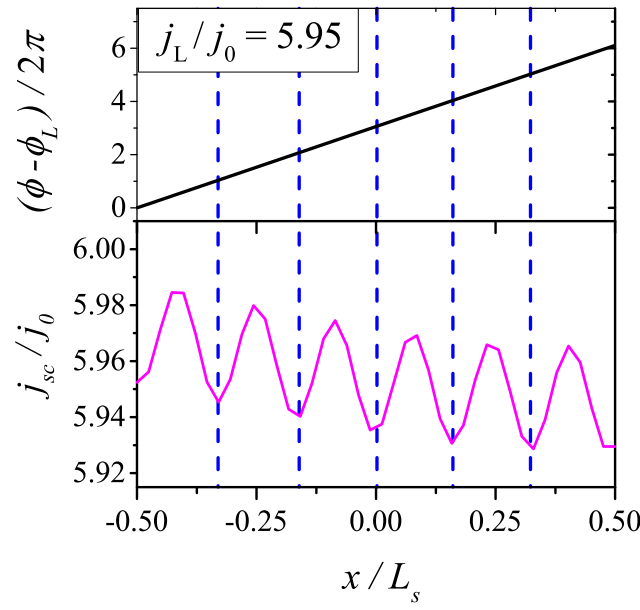

FIG. 2. For the six-vortex case $\left(j_{L} / j_{0}=5.95\right)$ when $d_{w}=0$. (Upper) Spatial profile of condensate phase relative to that at the left end $\phi-\phi_{L}$ (black solid line). The relative phase shows a straight line to bare eyes. The blue dashed lines indicate positions where the relative phase hits multiples of $2 \pi$. (Lower) The spatial profile of supercurrent density $j_{S C}$ (solid pink line). The supercurrent density essentially represents the phase gradient that allows the vortex contribution to stand out. Six full ripples in the pink line correspond to six vortices. The positions where $\phi-\phi_{L}=2 N \pi$ (blue dash line) coincide with the ripples' trough

are in fact the tunneling vortices governed by the sine-Gordon equation.

Mathematically, in the steady-state limit of the equation for $\dot{m}_{z}$ in Eq. (3), the equation is reduced to the stationary sineGordon equation (SsGE):

$$
\frac{\rho_{S}}{\hbar} \nabla^{2} \phi-\frac{1}{2} \frac{\Delta_{t} n}{\hbar} \sin \phi=0 \text {. }
$$

This equation is known to have a family of excited-state solutions, the sine-Gordon solitons, and each corresponds to a winding of multiples of $2 \pi$. Among them, the 1 soliton is the one with exactly $2 \pi$ phase winding. This 1 soliton shows a kink in the phase profile, which corresponds to a peak in the phase gradient profile. This peak is characterized by a length scale of

$$
\lambda=\sqrt{2 \rho_{s} / n \Delta_{t}},
$$

often referred to as the "Josephson length." Physically, we can identify the first term in Eq. (7), namely, $\left(\rho_{S} / \hbar\right) \nabla^{2} \phi$, as the gradient of supercurrent density, and the second term $\left(\Delta_{t} n / 2 \hbar\right) \sin \phi$ is the tunneling current (areal) density. That means that locally the supercurrent can turn into interlayer tunneling currents and reduce its magnitude. In the region where the condensate phase spans from 0 to $2 \pi$, the local tunneling current changes its direction from downward to upward accordingly, as shown in the lower panel of Fig. 1. The interplay of counterflow supercurrent and tunneling current forms a circular electron flow in this region. That is why we name the 1 soliton in our system a tunneling vortex.

When the external phase gradient is present (but not too large so as to induce a topological phase transition), the kink is then buried in the phase gradient, as is often seen in the flux-flow regime in the superconducting Josephson junction. 
That corresponds to a phase gradient profile showing only the tip of the original peak (which we refer to as "ripple" later). Now each vortex no longer claims a whole $\lambda$ space, but it can be more closely packed. That is what we observe in Fig. 2 .

In the supercurrent-density profile (lower panel of Fig. 2), we clearly identify six ripples, from trough to trough. The trough of these ripples lay on the blue dashed lines, marking the positions at which the exciton phase $\phi$ spans integer multiples of $2 \pi$. That is consistent with our understanding that for every $2 \pi$ phase span there exists a vortex. Since $j_{0}$ is the current density of adding an extra $2 \pi$ winding, it also adds a vortex to the system. That means that $j_{L} / j_{0}$ links exclusively to the vortex number in the system.

This vortex picture can be further examined by estimating the size of local current variation, the crest-to-trough difference of a ripple. From the current conservation discussed in the earlier paragraph, the loss in supercurrent has to transform into interlayer tunneling current. That loss, therefore, cannot exceed the maximum allowed areal tunneling current density en $\Delta_{t} /(2 \hbar)$ times half of the space a ripple claims, namely, half of $l_{\text {inter }} \equiv L_{s} / N$. That is,

$$
\frac{|\Delta j|}{j_{0}} \leqslant \frac{e n \Delta_{t} /(2 \hbar) \cdot l_{\text {inter }} / 2}{\left(e \rho_{S} / \hbar\right)\left(2 \pi / L_{S}\right)}=\frac{\bar{L}_{s}^{2}}{4 \pi N} .
$$

Here $\bar{L}_{s} \equiv L_{s} / \lambda$ is the dimensionless system length. Taking the parameters of Fig. 2, where $\bar{L}_{s}=1.6$ and $N=6$, the ripples then have a size of $|\Delta j| / j_{0} \sim 0.034$, while the read-off from Fig. 2 is about 0.035 . The good agreement supports our picture. Moreover, careful readers might also notice that the ripples sit on a background declining from left to right. That decline actually originates from the dynamic effect of tuning up the injected current at a finite increased rate and is beyond the sine-Gordon physics. We have found that by reducing the increased rate of the injected current, the decline becomes flattened.

We now discuss the injected-ejected current response (due to vortices) in the absence of weak condensate. Figure $3 \mathrm{com}-$ bines two plots: the ejected(injected) current density $j_{R}\left(j_{L}\right)$ [black solid (dash) lines] and the injected-ejected currentdensity difference $\Delta j$ vs $j_{L}$ [red solid line]. In both plots the blue dashed lines mark the $j_{L}$ with integer numbers of vortices; here the vortex numbers are obtained by counting the number of "ripples" in the current-density profiles at that $j_{L}$. These blue lines indeed fall in the close vicinity of integer multiples of $j_{L} / j_{0}$, justifying our assumption that the vortices are noninteracting. Next we find that $j_{R}$ and $j_{L}$ are almost indistinguishable to the naked eyes; their difference, $\Delta j \equiv$ $j_{R}-j_{L}$, is then plotted as a support. The $|\Delta j|$ shows wiggles with a typical amplitude of $\sim 0.05 j_{0}$, and the size of each wiggle is consistent with the estimation in Eq. (9). Moreover, we notice that $|\Delta j|$ drops almost to zero at integer numbers of vortices; the deviation from zero also originates from the dynamic effect of tuning up injected current. From the sineGordon physics, dropping to zero corresponds to having the crest on both the left and right ends in the current-density profile, and the $|\Delta j|$ comes from a net tunneling current that tilts the midpoints of the ripples, as seen in Fig. 2. When $j_{L} / j_{0}$ moves away from integers, the left end moves toward the crest and $|\Delta j|$ increases until $j_{L} / j_{0}$ is a half-integer. The left end is

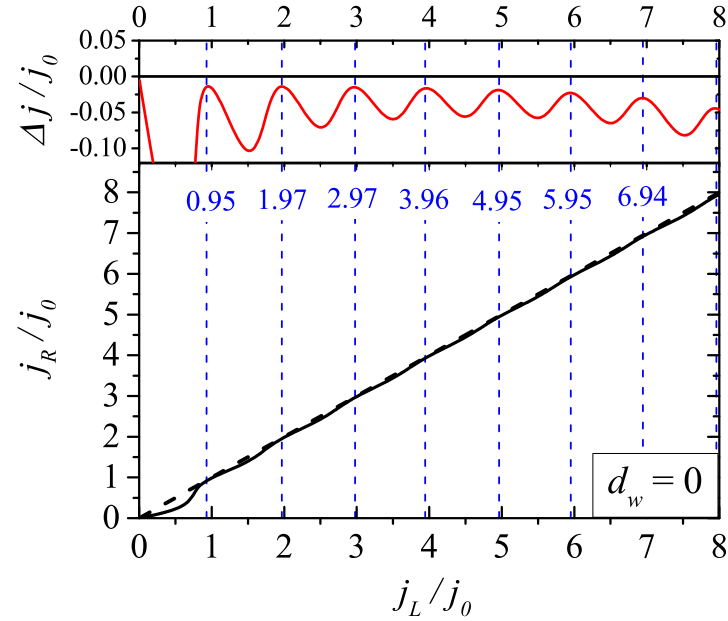

FIG. 3. The ejected $\left(j_{R}\right)$ vs injected current density $\left(j_{L}\right)$ (main panel) and $\Delta j$ vs $j_{L}$ (upper panel) when $d_{w}=0$. The solid black line represents the magnitude of the ejected $j_{R}$ while the injected $j_{L}$ (black dashed line) is also plotted for comparison. The $j_{L}$ values of which integer number of vortices occur are in blue dashed lines, which shows consistency with integer $j_{L} / j_{0}$ value. The injectedejected current-density difference $\Delta j$ is plotted in red in the upper panel and shows periods of roughly $j_{0}$ (one vortex) and amplitudes of around $0.05 j_{0}$

exactly at a crest while the right end is at a trough, and $|\Delta j|$ reaches its maximum with a value of roughly $\mathrm{t}_{s}^{2} / 4 \pi N$ [from Eq. (9)].

Up to this point, we have been focusing on the case of no weak condensate $\left(\mathrm{EC}^{\prime}\right)$. It turns out that the vortex effect can actually be enhanced in the presence of EC'. We first show the injected-ejected current response in this EC-EC'-EC system in Fig. 4; the same color scheme is used here as that in Fig. 3.

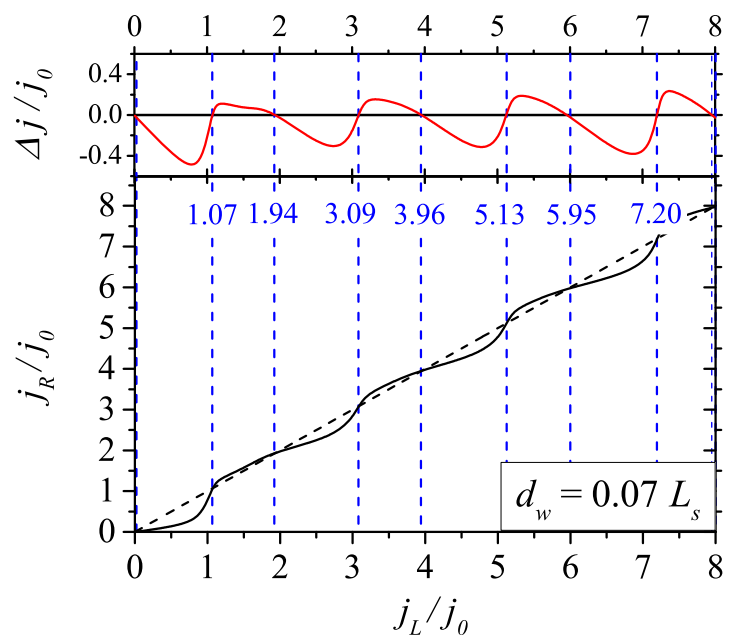

FIG. 4. The ejected $\left(j_{R}\right)$ vs injected current density $\left(j_{L}\right)$ (main panel) and $\Delta j$ vs $j_{L}$ (upper panel) when $d_{w}=0.07 L_{s}$. The coloring scheme is the same as that in Fig. 3. With finite $d_{w}, \Delta j$ is now greatly enhanced to hold amplitudes of roughly $0.5 j_{0}$. The value can be either positive or negative, meaning that the ejected current can exceed the injected. It is now with a periodicity of roughly $2 j_{0}$ (two vortices). 

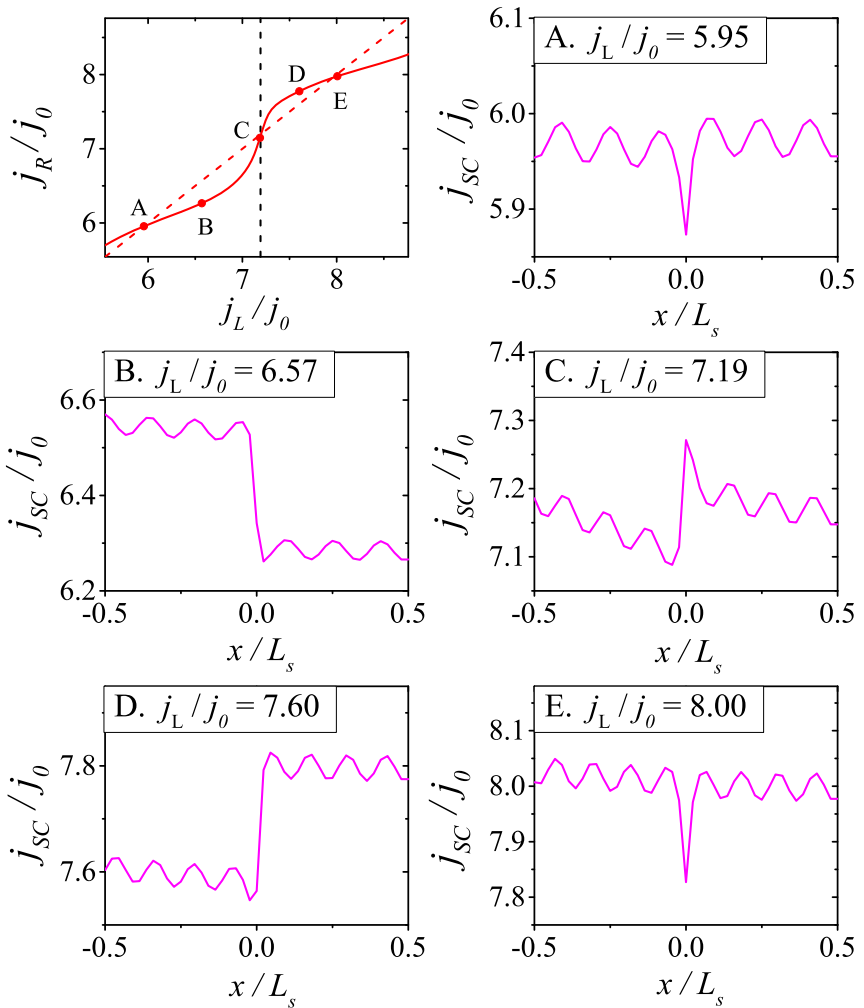

FIG. 5. Supercurrent-density profiles are taken between $j_{L} / j_{0}=$ 6 and 8 (one period in the $j_{L}$ vs $j_{R}$ plot). Point A corresponds to the case of precisely three vortices (ripples) in each EC. From point A to $\mathrm{C}$, the average supercurrent is larger in $\mathrm{EC} 1$ to facilitate vortex formation in that area; the number of vortices in EC2 stays the same. From point $\mathrm{C}$ to $\mathrm{D}$, the situation is the reverse. The supercurrent density in EC2 is higher than in EC1 to form vortices in the former. At point $\mathrm{E}$, one additional vortex is formed in each $\mathrm{EC}$, compared to point A.

Full vortices here still happen at around the integer $j_{L} / j_{0}$; the difference between $j_{R}$ and $j_{L}$, though, is noticeably enhanced in the EC-EC'-EC junction. The ejected current density $j_{R}$ still shows periodicity but now with a period of $2 j_{0}$. That comes with another unexpected feature that $j_{R}$ can go above $j_{L}$ in half of the period, while below in another half. This behavior is seen even more clearly in the $\Delta j$ curve in the same figure. When the floor of $j_{L} / j_{0}$ (vortex number) is odd, the difference $\Delta j$ is positive, whereas if the floor is even, $\Delta j$ turns negative.

The origin of the above feature is revealed when we inspect the supercurrent-density profiles taken over a period of $2 j_{0}$. Here we pick a typical period of $j_{L}=6 j_{0}$ to $8 j_{0}$ and look specifically at the five points marked A-E in Fig. 5(a). The number of vortices can be read off by counting the number of ripples. Note that the dip at the center originates from the larger capability of tunneling in the weak condensate. From A to $\mathrm{C}$, the ejected current is lower than that of the injected. Starting at $\mathrm{A}$, the injected current density $j_{L} / j_{0} \sim 6$, corresponding to six vortices in the condensate; when referring to the corresponding supercurrent-density profile, we do see three full vortices (from trough to trough) in each EC. As $j_{L}$ increases, $j_{S C}$ in the whole system increases accordingly, but more so in EC1 than in EC2. The larger average supercurrent
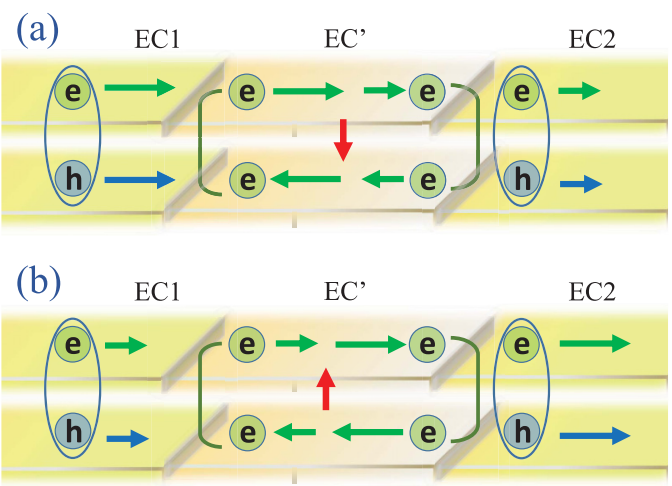

FIG. 6. Cartoon illustration of tunneling-assisted Andreev reflection (TAAR). The green (blue) arrows indicate the electron (hole) intralayer flows, and the red arrow shows the interlayer tunneling. (a) corresponds to B in Fig. 5 in which there is a net electron tunneling current from top to bottom so that the magnitude of the electron/hole current in the left EC/EC' boundary is more significant than that of the right. (b) corresponds to D in Fig. 5 with opposite tunneling current from that of B. The electron/hole current magnitudes are the reverse of that in (a).

in EC1 makes it easier to introduce an extra vortices in there, while the smaller average supercurrent in EC2 saves energy. Notice that this difference of supercurrent in EC1 and EC2 is unique to the EC/EC'/EC junction that does not have an analogy in the superconducting Josephson junction of the same geometry. In the superconducting Josephson junction, the Andreev reflection happens in both the left and right SC/N interface and conserves supercurrent. In our EC/EC'/EC junction, the tunneling-assisted Andreev reflection happens when EC' is long enough, as detailed in our previous work [38].

Here we illustrate in simple cartoon pictures as in Fig. 6. $\mathrm{B}$ in Fig. 5 corresponds to the cartoon in Fig. 6(a). Excitons (electron-hole, e-h, bound pair) Andreev-reflect at the left EC/EC' boundary (green curved line) and transform into electrons, flowing in opposite directions in the two layers. Part of the electrons in the top layer, however, cannot reach the right $\mathrm{EC} / \mathrm{EC}^{\prime}$ interface before going to the bottom layer through interlayer tunneling (red arrows). These electrons then flow back to the left EC/EC' interface, along with the other electrons originally in the bottom layer. This mechanism allows the supercurrents to be different between the left and right $\mathrm{EC} / \mathrm{EC}$ ' interface while the total current is still conserved at every point. When entering into the second half of the period (from $\mathrm{C}$ to $\mathrm{E}$ ), it saves more energy to do the opposite: introducing the additional vortex to EC2 instead of EC1. That corresponds to a larger average supercurrent in EC2 than in $\mathrm{EC} 1$, again through TAAR but in the opposite direction [as illustrated in Fig. 6(b)]. Over the $2 j_{0}$ period, we see that the presence of EC' enables the tunneling-assisted Andreev reflection and the average supercurrent in EC1 and EC2 can be freely tuned in an energy-saving way to introduce vorticesfirst in EC1 than in EC2. At the end of the period, the vortex numbers in both condensates are increased by 1 . Notice that the above-described TAAR is beyond sine-Gordon physics because when interlayer tunneling prefers one direction to the other, it involves charge imbalance. That leads to changes 

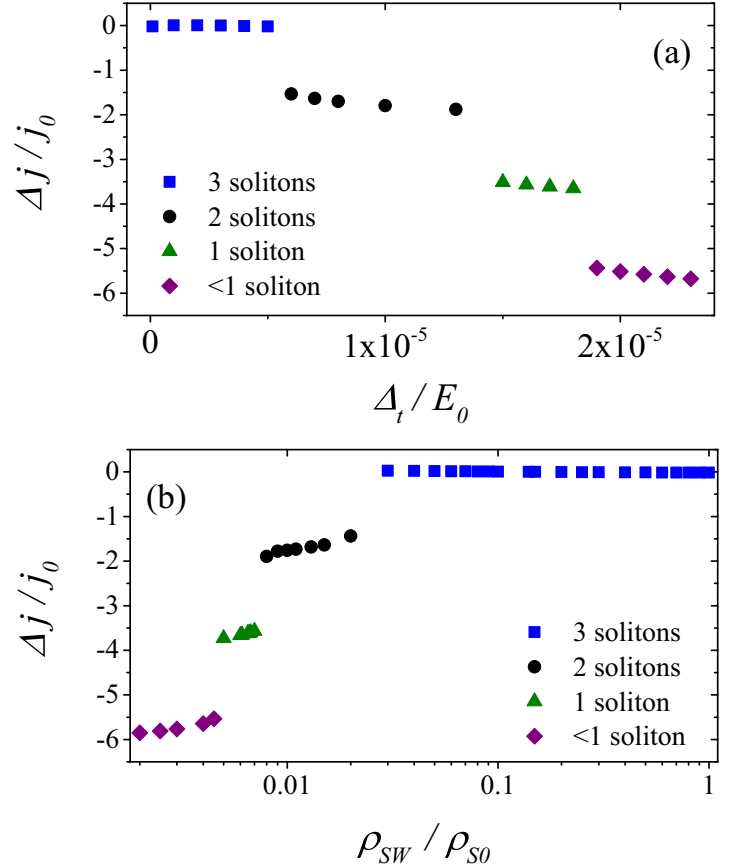

FIG. 7. For $j_{L} / j_{0}=5.95$, (a) $\Delta j$ vs tunneling strength $\Delta_{t}$ and (b) $\Delta j$ vs tunneling strength $\rho_{\mathrm{Sw}}$. Plateaus in $\Delta j$ are found to correspond to different numbers of vortices in the system.

in the pseudospin component $m_{z}$, which is not included in the sine-Gordon equation but is legitimately present in our original Landau-Lifshitz-Gilbert equation. It is important to note again that the current conservation is still fulfilled in the case and the total charge of the bilayer is conserved locally.

More interesting tunneling vortex effects are found when we vary other experimental controllable parameters. Figure 7 shows that the ejected-current responses to tunneling strength are actually quantized. In this figure we plot the injectedejected current difference $\Delta j$ versus the tunneling strength $\Delta_{t}$. The plot takes the same set of parameters as that in Fig. 5(a) (explicitly $j_{L}=5.95$ ), except for the tunneling strength $\Delta_{t}$ which varies in this plot. It turns out that although the tunneling strength varies continuously, the $\Delta j$ vs $\Delta_{t}$ curve breaks into plateaus. Data points in the same plateau then share the same number of vortices. Explicitly from small to large $\Delta_{t}$, squares at the first plateau correspond to three vortices (blue), the second to two vortices (black), the third to one vortex (green), and the fourth to none (purple) in EC2 alone. This means that when the interlayer tunneling increases, the current drop due to TAAR increases-most importantly, in a quantized fashion. If we model the drop as the current reduction in $\mathrm{EC} 2$ when reducing one vortex in $\mathrm{EC} 2$ (of length roughly $L_{s} / 2$ ), each of them should have a size of

$$
\frac{e \rho_{S}}{\hbar} \frac{2 \pi}{L_{S} / 2} \sim 2 j_{0} \text {. }
$$

This is again consistent with value found in Fig. 7(a). Please note that although the plot is for a particular $j_{L}$ (point A), the quantization behavior is, in fact, general that occurs at all $j_{L}$.
A similar quantization behavior is also found in the ejected-current response to the superfluid density at EC', $\rho_{\mathrm{SW}}$. In Fig. $7(\mathrm{~b}), \Delta j$ vs $\rho_{\mathrm{SW}}$ also at point $\mathrm{A}\left(j_{L} / j_{0}=5.95\right)$ shows ascending plateaus that correspond to less than one (purple), one (green), two (black), and three (blue) vortices, from small to large $\rho_{\mathrm{SW}}$. The jump between every two plateaus is again $2 j_{0}$, which can be argued exactly like that for tunneling strength. Finally, we note that out of the two quantities, $\Delta_{t}$ and $\rho_{\mathrm{SW}}$, the latter can be a more convenient knob for experiments. The reason is that a bilayer qualified for condensation usually has a tunneling strength of $10^{-6}$ or less, which gives a relatively smaller room to play around. The superfluid density $\rho_{\mathrm{SW}}$, on the other hand, can be adjusted more easily and over a broader range by depleting the electron density in one of the layers. We therefore believe that the vortices' quantization behavior has a great potential to be observed through the $\rho_{\mathrm{SW}}$ adjustment.

In the end, we would like to comment on related work by Eastham et al. [71,72]. In their work, Eastham et al. focus on a randomly disordered but homogeneously structured excitonic bilayer. Because of the homogeneity in structure, they were able to consider the superfluid phase alone and obtained a sine-Gordon-like equation even under their disorder percolating network model. In our calculation, though, the EC/EC'/EC structure makes it necessary to consider the $z$ component of pseudospin. In fact, that is also the origin of the intriguing phenomena that we report.

\section{SUMMARY}

We demonstrate how the current response can manifest the quantization of tunneling vortices in the current-injection scheme. In an excitonic condensate, tunneling vortices, induced by interlayer tunneling, cause the ejected current to differ from the injected current. The injected-ejected current difference shows a periodicity for every occurrence of one vortex. This difference is even more profound when inserting a weak condensate (EC') to the junction. In such junctions (EC/EC'/EC), the tunneling-assisted Andreev reflection allows the average supercurrent to differ in EC1 and EC2. The injected-ejected current difference then shows a periodicity for each occurrence of two vortices. The quantized nature of vortices also appears when fixing the injected current and changing the tunneling strength $\Delta_{t}$ or the superfluid density in the weak condensate, $\rho_{\mathrm{SW}}$. The injected-ejected current difference shows plateaus when tuning the above two parameters. The manifestation of that quantization behavior is particularly viable experimentally.

\section{ACKNOWLEDGMENTS}

We are grateful to A. H. MacDonald, J. P. Eisenstein, and Chien-Te Wu for valuable discussions. The authors were supported by the Ministry of Science and Technology (MOST 108-2811-M-009-557 for Y.F.H. and MOST 110-2112-MA49-011-, MOST 106-2112-M-009-011-MY3 for all other authors). 
[1] S. M. Girvin and A. H. MacDonald, in Perspectives in Quantum Hall Effects, edited by S. Das Sarma and A. Pinczuk (Wiley, New York, 1997), Chap. V; J. P. Eisenstein, ibid., Chap. II.

[2] J. P. Eisenstein, Exciton condensation in bilayer quantum Hall systems, Annu. Rev. Condens. Matter Phys. 5, 159 (2014).

[3] L. V. Keldysh and A. N. Kozlov, Collective properties of excitons in semiconductors, Sov. Phys. JETP 27, 521 (1968).

[4] I. B. Spielman, J. P. Eisenstein, L. N. Pfeiffer, and K. W. West, Resonantly Enhanced Tunneling in a Double Layer Quantum Hall Ferromagnet, Phys. Rev. Lett. 84, 5808 (2000); Observation of a Linearly Dispersing Collective Mode in a Quantum Hall Ferromagnet, 87, 036803 (2001); I. B. Spielman, M. Kellogg, J. P. Eisenstein, L. N. Pfeiffer, and $\mathrm{K}$. W. West, Onset of interlayer phase coherence in a bilayer two-dimensional electron system: Effect of layer density imbalance, Phys. Rev. B 70, 081303(R) (2004); I. B. Spielman, L. A. Tracy, J. P. Eisenstein, L. N. Pfeiffer, and K. W. West, Spin Transition in Strongly Correlated Bilayer Two-Dimensional Electron Systems, Phys. Rev. Lett. 94, 076803 (2005).

[5] A. R. Champagne, J. P. Eisenstein, L. N. Pfeiffer, and K. W. West, Evidence for a Finite-Temperature Phase Transition in a Bilayer Quantum Hall System, Phys. Rev. Lett. 100, 096801 (2008); A. R. Champagne, A. D. K. Finck, J. P. Eisenstein, L. N. Pfeiffer, and K. W. West, Charge imbalance and bilayer two-dimensional electron systems at $v_{T}=1$, Phys. Rev. B 78, 205310 (2008).

[6] A. D. K. Finck, A. R. Champagne, J. P. Eisenstein, L. N. Pfeiffer, and K. W. West, Area dependence of interlayer tunneling in strongly correlated bilayer two-dimensional electron systems at $v_{T}=1$, Phys. Rev. B 78, 075302 (2008).

[7] L. Tiemann, W. Dietsche, M. Hauser, and K. von Klitzing, Critical tunneling currents in the regime of bilayer excitons, New J. Phys. 10, 045018 (2008); L. Tiemann, Y. Yoon, W. Dietsche, K. von Klitzing, and W. Wegscheider, Dominant parameters for the critical tunneling current in bilayer exciton condensates, Phys. Rev. B 80, 165120 (2009).

[8] X. Huang, W. Dietsche, M. Hauser, and K. von Klitzing, Coupling of Josephson Currents in Quantum Hall Bilayers, Phys. Rev. Lett. 109, 156802 (2012).

[9] T. Hyart and B. Rosenow, Influence of Topological Excitations on Shapiro Steps and Microwave Dynamical Conductance in Bilayer Exciton Condensates, Phys. Rev. Lett. 110, 076806 (2013).

[10] D. Nandi, T. Khaire, A. D. K. Finck, J. P. Eisenstein, L. N. Pfeiffer, and K. W. West, Tunneling at $v_{T}=1$ in quantum Hall bilayers, Phys. Rev. B 88, 165308 (2013).

[11] M. Kellogg, I. B. Spielman, J. P. Eisenstein, L. N. Pfeiffer, and K. W. West, Observation of Quantized Hall Drag in a Strongly Correlated Bilayer Electron System, Phys. Rev. Lett. 88, 126804 (2002); M. Kellogg, J. P. Eisenstein, L. N. Pfeiffer, and K. W. West, Bilayer Quantum Hall Systems at $v_{T}=1$ : Coulomb Drag and the Transition from Weak to Strong Interlayer Coupling, ibid. 90, 246801 (2003).

[12] E. Tutuc, S. Melinte, E. P. De Poortere, R. Pillarisetty, and M. Shayegan, Role of Density Imbalance in an Interacting Bilayer Hole System, Phys. Rev. Lett. 91, 076802 (2003); E. Tutuc, R. Pillarisetty, and M. Shayegan, Giant frictional drag in strongly interacting bilayers near filling factor one, Phys. Rev. B 79, 041303(R) (2009).

[13] R. D. Wiersma, J. G. S. Lok, S. Kraus, W. Dietsche, K. von Klitzing, D. Schuh, M. Bichler, H. P. Tranitz, and W. Wegscheider, Activated Transport in the Separate Layers that Form the $v_{T}=1$ Exciton Condensate, Phys. Rev. Lett. 93, 266805 (2004).

[14] A. D. K. Finck, J. P. Eisenstein, L. N. Pfeiffer, and K. W. West, Quantum Hall Exciton Condensation at Full Spin Polarization, Phys. Rev. Lett. 104, 016801 (2010).

[15] J.-J. Su and A. H. MacDonald, How to make a bilayer exciton condensate flow, Nat. Phys. 4, 799 (2008).

[16] D. Nandi, A. D. K. Finck, J. P. Eisenstein, L. N. Pfeiffer, and K. W. West, Exciton condensation and perfect Coulomb drag, Nature (London) 488, 481 (2012).

[17] K. Yang, K. Moon, L. Zheng, A. H. MacDonald, S. M. Girvin, D. Yoshioka, and S.-C. Zhang, Quantum Ferromagnetism and Phase Transitions in Double-Layer Quantum Hall Systems, Phys. Rev. Lett. 72, 732 (1994).

[18] K. Moon, H. Mori, K. Yang, S. M. Girvin, A. H. MacDonald, L. Zheng, D. Yoshioka, and S.-C. Zhang, Spontaneous interlayer coherence in double-layer quantum Hall systems: Charged vortices and Kosterlitz-Thouless phase transitions, Phys. Rev. B 51, 5138 (1995).

[19] K. Yang, K. Moon, L. Belkhir, H. Mori, S. M. Girvin, A. H. MacDonald, L. Zheng, and D. Yoshioka, Spontaneous interlayer coherence in double-layer quantum Hall systems: Symmetry-breaking interactions, in-plane fields, and phase solitons, Phys. Rev. B 54, 11644 (1996).

[20] A. A. Burkov and A. H. MacDonald, Lattice pseudospin model for $v=1$ quantum Hall bilayers, Phys. Rev. B 66, 115320 (2002).

[21] R. V. Gorbachev, A. K. Geim, M. I. Katsnelson, K. S. Novoselov, T. Tudorovskiy, I. V. Grigorieva, A. H. MacDonald, S. V. Morozov, K. Watanabe, T. Taniguchi, and L. A. Ponomarenko, Strong Coulomb drag and broken symmetry in double-layer graphene, Nat. Phys. 8, 896 (2012).

[22] A. Perali, D. Neilson, and A. R. Hamilton, High-Temperature Superfluidity in Double-Bilayer Graphene, Phys. Rev. Lett. 110, 146803 (2013).

[23] M. Titov, R. V. Gorbachev, B. N. Narozhny, T. Tudorovskiy, M. Schütt, P. M. Ostrovsky, I. V. Gornyi, A. D. Mirlin, M. I. Katsnelson, K. S. Novoselov, A. K. Geim, and L. A. Ponomarenko, Giant Magnetodrag in Graphene at Charge Neutrality, Phys. Rev. Lett. 111, 166601 (2013).

[24] J. I. A. Li, T. Taniguchi, K. Watanabe, J. Hone, A. Levchenko, and C. R. Dean, Negative Coulomb Drag in Double Bilayer Graphene, Phys. Rev. Lett. 117, 046802 (2016).

[25] K. Lee, J. Xue, D. C. Dillen, K. Watanabe, T. Taniguchi, and E. Tutuc, Giant Frictional Drag in Double Bilayer Graphene Heterostructures, Phys. Rev. Lett. 117, 046803 (2016).

[26] J.-J. Su and A. H. MacDonald, Spatially indirect exciton condensate phases in double bilayer graphene, Phys. Rev. B 95 045416 (2017).

[27] X. Liu, K. Watanabe, T. Taniguchi, B. I. Halperin, and P. Kim, Quantum Hall drag of exciton condensate in graphene, Nat. Phys. 13, 746 (2017). 
[28] J. I. A. Li, T. Taniguchi, K. Watanabe, J. Hone, and C. R. Dean, Excitonic superfluid phase in double bilayer graphene, Nat. Phys. 13, 751 (2017).

[29] J. I. A. Li, Q. Shi, Y. Zeng, K. Watanabe, T. Taniguchi, J. Hone, and C. R. Dean, Pairing states of composite fermions in doublelayer graphene, Nat. Phys. 15, 898 (2019).

[30] B. A. Malomed, The Sine-Gordon model: General background, physical motivations, inverse scattering, and solitons, in The Sine-Gordon Model and It's Applications, edited by J. Cuevas-Maraver, P. G. Kevrekidis, and F. Williams (Springer International Publishing, Cham, Switzerland, 2014), pp. 1-30; J. J. Mazo and A. V. Ustinov, The sine-Gordon equation in Josephson-junction arrays, ibid., pp. 155-176.

[31] See, for example, L. Q. English, Experimental results for the sine-Gordon equation in arrays of coupled torsion pendula, in The Sine-Gordon Model and Its Applications, edited by J. Cuevas-Maraver, P. G. Kevrekidis, and F. Williams (Springer International Publishing, Cham, Switzerland, 2014).

[32] X. G. Wen and A. Zee, Sideways tunnelling and fractional Josephson frequency in double-layered quantum Hall systems, Europhys. Lett. 35, 227 (1996).

[33] M. Abolfath, A. H. MacDonald, and L. Radzihovsky, Critical currents of ideal quantum Hall superfluids, Phys. Rev. B 68, 155318 (2003).

[34] Y. N. Joglekar, A. V. Balatsky, and M. P. Lilly, Excitonic condensate and quasiparticle transport in electron-hole bilayer systems, Phys. Rev. B 72, 205313 (2005).

[35] K. Park and S. Das Sarma, Coherent tunneling in exciton condensates of bilayer quantum Hall systems, Phys. Rev. B 74, 035338 (2006).

[36] J.-J. Su and A. H. MacDonald, Critical tunneling currents in quantum Hall superfluids: Pseudospin-transfer torque theory, Phys. Rev. B 81, 184523 (2010).

[37] Y.-F. Hsu and J.-J. Su, Fractional solitons in excitonic Josephson junctions, Sci. Rep. 5, 15796 (2015).

[38] Y.-F. Hsu and J.-J. Su, Single interface effects dominate in exciton-condensate/normal-barrier/exciton-condensate (EC/N/EC) structures of long-barrier, New J. Phys. 20, 083002 (2018).

[39] Y.-F. Hsu and J.-J. Su, Excitonic Josephson effect induced by interlayer tunneling current: Robust evidence for exciton condensation, arXiv:2006.15329.

[40] E. Goldobin, A. Sterck, T. Gaber, D. Koelle, and R. Kleiner, Dynamics of Semifluxons in Nb Long Josephson 0- $\pi$ Junctions, Phys. Rev. Lett. 92, 057005 (2004).

[41] E. Goldobin, D. Koelle, and R. Kleiner, Ground states of one and two fractional vortices in long Josephson $0-\kappa$ junctions, Phys. Rev. B 70, 174519 (2004).

[42] K. Buckenmaier, T. Gaber, M. Siegel, D. Koelle, R. Kleiner, and E. Goldobin, Spectroscopy of the Fractional Vortex Eigenfrequency in a Long Josephson 0- $\kappa$ Junction, Phys. Rev. Lett. 98, 117006 (2007).

[43] H. Sickinger, A. Lipman, M. Weides, R. G. Mints, H. Kohlstedt, D. Koelle, R. Kleiner, and E. Goldobin, Experimental Evidence of a $\varphi$ Josephson Junction, Phys. Rev. Lett. 109, 107002 (2012).

[44] D. M. Heim, K. Vogel, W. P. Schleich, D. Koelle, R. Kleiner, and E. Goldobin, A tunable macroscopic quantum system based on two fractional vortices, New J. Phys. 15, 053020 (2013).
[45] D. B. Szombati, S. Nadj-Perge, D. Car, S. R. Plissard, E. P. A. M. Bakkers, and L. P. Kouwenhoven, Josephson $\phi_{0^{-}}$ junction in nanowire quantum dots, Nat. Phys. 12, 568 (2016).

[46] G. Grüner, Density Waves in Solids (Addison-Wesley, Reading, MA, 1994), and references therein.

[47] P. Bak and V. L. Pokrovsky, Theory of Metal-Insulator Transition in Peierls Systems with Nearly Half-Filled Bands, Phys. Rev. Lett. 47, 958 (1981)

[48] T. Nagatsuma, K. Enpuku, F. Irie, and K. Yoshida, Flux-flow type Josephson oscillator for millimeter and submillimeter wave region, J. Appl. Phys. 54, 3302 (1983).

[49] A. V. Ustinov, J. Mygind, and V. A. Oboznov, Phase-locked flux-flow Josephson oscillator, J. Appl. Phys. 72, 1203 (1992).

[50] V. P. Koshelets and S. V. Shitov, Integrated superconducting receivers, Supercond. Sci. Technol. 13, R53 (2000).

[51] V. P. Koshelets, S. V. Shitov, P. N. Dmitriev, A. B. Ermakov, L. V. Filippenko, V. V. Khodos, V. L. Vaks, A. M. Baryshev, P. R. Wesselius, and J. Mygind, Towards a phase-locked superconducting integrated receiver: Prospects and limitations, Physica C 367, 249 (2002).

[52] M. Y. Torgashin, V. P. Koshelets, P. N. Dmitriev, A. B. Ermakov, L. V. Filippenko, and P. A. Yagoubov, Superconducting integrated receiver based on $\mathrm{Nb}-\mathrm{AlN}-\mathrm{NbN}-\mathrm{Nb}$ circuits, IEEE Trans. Appl. Supercond. 17, 379 (2007).

[53] T. Nagatsuma, K. Enpuku, K. Sueoka, K. Yoshida, and F. Irie, Flux-flow-type Josephson oscillator for millimeter and submillimeter wave region. III. Oscillation stability, J. Appl. Phys. 58, 441 (1985).

[54] A. A. Golubov, B. A. Malomed, and A. V. Ustinov, Radiation linewidth of a long Josephson junction in the flux-flow regime, Phys. Rev. B 54, 3047 (1996).

[55] A. L. Pankratov, Form and width of the spectral line of a Josephson flux-flow oscillator, Phys. Rev. B 65, 054504 (2002).

[56] J. Mygind, V. P. Koshelets, M. R. Samuelsen, and A. S. Sobolev, The submm wave Josephson flux flow oscillator, Linewidth measurements and simple theory, IEEE Trans. Appl. Supercond. 15, 968 (2005).

[57] A. L. Pankratov, V. L. Vaks, and V. P. Koshelets, Spectral properties of phase-locked flux flow oscillator, J. Appl. Phys. 102, 063912 (2007).

[58] A. L. Pankratov, Minimizing the linewidth of the flux-flow oscillator, Appl. Phys. Lett. 92, 082504 (2008).

[59] D. R. Gulevich, V. P. Koshelets, and F. V. Kusmartsev, Josephson flux-flow oscillator: The microscopic tunneling approach, Phys. Rev. B 96, 024515 (2017).

[60] A. V. Ustinov, Fluxon insertion into annular Josephson junctions, Appl. Phys. Lett. 80, 3153 (2002).

[61] M. Beck, E. Goldobin, M. Neuhaus, M. Siegel, R. Kleiner, and D. Koelle, High-Efficiency Deterministic Josephson Vortex Ratchet, Phys. Rev. Lett. 95, 090603 (2005).

[62] K. G. Fedorov, A. V. Shcherbakova, M. J. Wolf, D. Beckmann, and A. V. Ustinov, Fluxon Readout of a Superconducting Qubit, Phys. Rev. Lett. 112, 160502 (2014).

[63] B. A. Malomed and A. V. Ustinov, Creation of classical and quantum fluxons by a current dipole in a long Josephson junction, Phys. Rev. B 69, 064502 (2004).

[64] E. Goldobin, N. Stefanakis, D. Koelle, and R. Kleiner, Fluxonsemifluxon interaction in an annular long Josephson $0-\pi$ junction, Phys. Rev. B 70, 094520 (2004). 
[65] A. Fedorov, A. Shnirman, and G. Schön, and A. KidiyarovaShevchenko, Reading out the state of a flux qubit by Josephson transmission line solitons, Phys. Rev. B 75, 224504 (2007).

[66] D. R. Gulevich, F. V. Kusmartsev, S. Savel'ev, V. A. Yampol'skii, and F. Nori, Shape Waves in 2D Josephson Junctions: Exact Solutions and Time Dilation, Phys. Rev. Lett. 101, 127002 (2008).

[67] A. Dua, B. Malomed, M. Cheng, and L. Jiang, Universal quantum computing with parafermions assisted by a half-fluxon, Phys. Rev. B 100, 144508 (2019).

[68] E. Rossi, A. S. Nunez, and A. H. MacDonald, Interlayer Transport in Bilayer Quantum Hall Systems, Phys. Rev. Lett. 95, 266804 (2005).
[69] T. Hyart and B. Rosenow, Quantitative description of Josephson-like tunneling in $v_{T}=1$ quantum Hall bilayers, Phys. Rev. B 83, 155315 (2011).

[70] S. Q. Murphy, J. P. Eisenstein, L. N. Pfeiffer, and K. W. West, Lifetime of two-dimensional electrons measured by tunneling spectroscopy, Phys. Rev. B 52, 14825 (1995).

[71] P. R. Eastham, N. R. Cooper, and D. K. K. Lee, Critical Supercurrents and Self-Organization in Quantum Hall Bilayers, Phys. Rev. Lett 105, 236805 (2010).

[72] P. R. Eastham, N. R. Cooper, and D. K. K. Lee, Diamagnetism and flux creep in bilayer exciton superfluids, Phys. Rev. B 85, 165320 (2012). 